\title{
AN IMPEDANCE EFFECT OF A THIN ADHESIVE LAYER IN SOME BOUNDARY VALUE AND TRANSMISSION PROBLEMS GOVERNED BY ELLIPTIC DIFFERENTIAL EQUATIONS
}

A. Favini, University of Bologna, Department of Mathematics, Bologna, Italy, favini@dm.unibo.it,

R. Labbas, Laboratory of Mathematics Applied, University of du Havre, Le Havre, France, Rabah.Labbas@univ-lehavre.fr,

K. Lemrabet, Laboratory AMNEDP, Faculty of Mathematics, University of Sciences and Technology - Houari Boumediene, Algiers, Algeria, keddourlemrabet@yahoo.fr

In this paper we consider a problem of two bodies bonded through a thin adhesive layer (a third material) of thickness $\delta$. Leting $\delta$ go to zero, one obtains a boundary value transmission problem set on a fixed domain. We then give new results for the study of this problem in the framework of Hölder spaces: an explicit representation of the solution and necessary and sufficient conditions at the interface for its optimal regularity are obtained using the semigroups theory and the real interpolation spaces.

Keywords: boundary value problem of elliptic type; transmission problems; impedance effect; thin layer.

To the memory of Alfredo Lorenzi.

\section{Introduction}

Consider the boundary value and transmission second-order operational problem

$$
\left(P_{\delta}\right)\left\{\begin{array}{l}
\left.\left(u^{\delta}\right)^{\prime \prime}(x)+A u^{\delta}(x)=g^{\delta}(x) \quad \text { on }\right]-1,0[\cup] 0, \delta[\cup] \delta, 1+\delta[ \\
u^{\delta}(-1)=f_{-} \\
\left(u^{\delta}\right)^{\prime}(1+\delta)=f_{+} \\
u^{\delta}\left(0^{-}\right)=u^{\delta}\left(0^{+}\right)+\alpha^{\delta}, u^{\delta}\left(\delta^{-}\right)=u^{\delta}\left(\delta^{+}\right)+\beta^{\delta} \\
p_{-}\left(u^{\delta}\right)^{\prime}\left(0^{-}\right)=p_{0}\left(u^{\delta}\right)^{\prime}\left(0^{+}\right)+a^{\delta} \\
p_{0}\left(u^{\delta}\right)^{\prime}\left(\delta^{-}\right)=p_{+}\left(u^{\delta}\right)^{\prime}\left(\delta^{+}\right)+b^{\delta}
\end{array}\right.
$$

set in some complex Banach space $E$; here $A$ is a closed linear operator of domain $D(A) \subset$ $E$ (not necessarily dense in $E$ ) which verifies the Krein's ellipticity condition (see Section 2, (16)) $f_{-}, f_{+}, \alpha^{\delta}, \beta^{\delta}, a^{\delta}, b^{\delta}$ are given in $E$ and satisfy some necessary and sufficient conditions which will be specified later.

The function $g^{\delta}$ is such that

$$
\left\{\begin{array}{l}
g_{-}=\left.g^{\delta}\right|_{[-1,0]} \in C^{\eta}([-1,0] ; E) \\
g_{0}^{\delta}=\left.g^{\delta}\right|_{[0, \delta]} \in C^{\eta}([0, \delta] ; E) \\
g_{+}^{\delta}=\left.g^{\delta}\right|_{[\delta, 1+\delta]} \in C^{\eta}([\delta, 1+\delta] ; E),
\end{array}\right.
$$

(with $0<\eta<1$ ). It is not difficult to prove that the hölderianity of $g_{-}, g_{0}^{\delta}$ and $g_{+}^{\delta}$ imply the global hölderianity of $g^{\delta}$ on $[-1,1+\delta]$ if and only if 


$$
g_{-}(0)=g_{0}^{\delta}(0) \quad \text { and } \quad g_{0}^{\delta}(\delta)=g_{+}^{\delta}(\delta) .
$$

We do not assume these two conditions.

Set

$$
u_{-}^{\delta}=u_{\mid]-1,0[}^{\delta}, \quad u_{0}^{\delta}=u_{\mid] 0, \delta[}^{\delta}, \quad u_{+}^{\delta}=u_{\mid] \delta, 1+\delta[}^{\delta},
$$

then problem $\left(P_{\delta}\right)$ writes

$$
\left(P_{\delta}\right)\left\{\begin{aligned}
(E Q S)\left\{\begin{array}{l}
\left.\left(u_{-}^{\delta}\right)^{\prime \prime}(x)+A u_{-}^{\delta}(x)=g_{-}(x) \text { on }\right]-1,0[ \\
\left.\left(u_{0}^{\delta}\right)^{\prime \prime}(x)+A u_{0}^{\delta}(x)=g_{0}^{\delta}(x) \text { on }\right] 0, \delta[ \\
\left.\left(u_{+}^{\delta}\right)^{\prime \prime}(x)+A u_{+}^{\delta}(x)=g_{+}^{\delta}(x) \text { on }\right] \delta, 1+\delta[,
\end{array}\right. \\
(B C)\left\{\begin{array}{l}
u_{-}^{\delta}(-1)=f_{-} \\
\left(u_{+}^{\delta}\right)^{\prime}(1+\delta)=f_{+},
\end{array}\right. \\
\left\{\begin{array}{l}
u_{-}^{\delta}(0)=u_{0}^{\delta}(0)+\alpha^{\delta} \\
u_{0}^{\delta}(\delta)=u_{+}^{\delta}(\delta)+\beta^{\delta} \\
p_{-}\left(u_{-}^{\delta}\right)^{\prime}(0)=p_{0}\left(u_{0}^{\delta}\right)^{\prime}(0)+a^{\delta} \\
p_{0}\left(u_{0}^{\delta}\right)^{\prime}(\delta)=p_{+}\left(u_{+}^{\delta}\right)^{\prime}(\delta)+b^{\delta} .
\end{array}\right.
\end{aligned}\right.
$$

The numerical solution of this problem is usually very difficult to compute. In fact, the small thickness of the thin layer generates difficulties in the meshing. As $\delta \rightarrow 0$, the interval $] 0, \delta[$ degenerates into the point $\{0\}$ and we can no more have an equation on it. The interval $] \delta, \delta+1[$ becomes $] 0,1[$.

Therefore, the main question is: what will be the appropriate transmission conditions at the interface point $\{0\}$ which describe correctly the effect of the thin adhesive layer ] $0, \delta[$ as $\delta \rightarrow 0 ?$

We will answer formally to this question in the most interesting case, characterizing so the effect of the small bond $] 0, \delta[$ (as $\delta \rightarrow 0)$.

Let us begin by giving a formal derivation of the effect of the small bond $] 0, \delta[$ (as $\delta \rightarrow 0)$.

In order to deal with our problem $\left(P_{\delta}\right)$, we solve the scalar equation on the small interval $] 0, \delta[$ and write down relations between the Cauchy data

$$
\left(u_{0}^{\delta}(0),\left(u_{0}^{\delta}\right)^{\prime}(0)\right) \text { and }\left(u_{0}^{\delta}(\delta),\left(u_{0}^{\delta}\right)^{\prime}(\delta)\right) .
$$

Then making use of the transmission condition at $\{0\}$ and $\{\delta\}$, we will deduce relations linking

$$
\left(u_{-}^{\delta}(0), p_{-}\left(u_{-}^{\delta}\right)^{\prime}(0)\right)
$$

to

$$
\left(u_{+}^{\delta}(\delta), p_{+}\left(u_{+}^{\delta}\right)^{\prime}(\delta)\right)
$$

which allow us, as $\delta \rightarrow 0$, to obtain the limiting transmission conditions at $\{0\}$. Therefore, we will see that the interesting limit problem writes in the form

$$
\left(P_{1 A}\right)\left\{\begin{array}{l}
\left.\left(u_{-}\right)^{\prime \prime}(x)+A u_{-}(x)=g_{-}(x) \quad \text { on }\right]-1,0[ \\
\left.\left(w_{+}\right)^{\prime \prime}(x)+A w_{+}(x)=h_{+}(x) \quad \text { on }\right] 0,1[ \\
u(-1)=f_{-},\left(w_{+}\right)^{\prime}(1)=f_{+} \\
u_{-}(0)=w_{+}(0)+\varphi \\
p_{-}\left(u_{-}\right)^{\prime}(0)-p_{+}\left(w_{+}\right)^{\prime}(0)=q A u_{-}(0)+\psi,
\end{array}\right.
$$


see the details in Subsection 1.1.

Our main results concerning problem $\left(P_{1 A}\right)$ are summarized in the following Theorem.

Theorem 1. Let $g_{-} \in C^{\eta}([-1,0] ; E), h_{+} \in C^{\eta}([0,1] ; E)$ with $0<\eta<1$ and $f_{-} \in D(A)$, $f_{+} \in D\left((-A)^{1 / 2}\right), \varphi \in D(A), \psi \in E$. Assume (16), see below. Then problem $\left(P_{1 A}\right)$ has a unique solution

$$
\left\{\begin{array}{l}
\left.u_{-}(x):\right]-1,0[\rightarrow E \\
\left.w_{+}(x):\right] 0,1[\rightarrow E
\end{array}\right.
$$

such that

1. $u_{-} \in C([-1,0] ; D(A)) \cap C^{2}([-1,0] ; E), w_{+} \in C([0,1] ; D(A)) \cap C^{2}([0,1] ; E)$ if and only if

$$
\left\{\begin{array}{l}
(* s)\left\{\begin{array}{l}
A f_{-}-g_{-}(-1) \in \overline{D(A)} \\
(-A)^{1 / 2} f_{+} \in \overline{D(A)}
\end{array}\right. \\
(* * s)\left\{\begin{array}{l}
q g_{-}(0)+\psi \in \overline{D(A)} \\
h_{+}(0)-g_{-}(0)+A \varphi \in \overline{D(A)}
\end{array}\right.
\end{array}\right.
$$

2. $A u_{-}(),. u_{-}^{\prime \prime} \in C^{\eta}([-1,0] ; E), A w_{+}(),. w_{+}^{\prime \prime} \in C^{\eta}([0,1] ; E)$ if and only if

$$
\left\{\begin{array}{l}
(* r)\left\{\begin{array}{l}
A f_{-}-g_{-}(-1) \in D_{A}(\eta / 2,+\infty) \\
(-A)^{1 / 2} f_{+} \in D_{A}(\eta / 2,+\infty)
\end{array}\right. \\
(* * r)\left\{\begin{array}{l}
q g_{-}(0)+\psi \in D_{A}(\eta / 2,+\infty) \\
h_{+}(0)-g_{-}(0)+A \varphi \in D_{A}(\eta / 2,+\infty) .
\end{array}\right.
\end{array}\right.
$$

In this main result, note that $(* s)$ and $(* * s)$ are respectively the necessary and sufficient compatibility conditions at the boundary and the necessary and sufficient compatibility conditions at the interface $\{0\}$ to obtain a strict solution $u=\left(u_{-}, w_{+}\right)$. Similarly, $(* r)$ and $(* * r)$ are those to obtain optimal regularities on $u$.

The definition and the properties of the interpolation space $D_{A}(\eta / 2,+\infty)$ are given, for instance, in ( [1])

Many authors have worked on analogous problems, see [2-4] in hilbertian spaces. In $[5,6]$, a study is given for a similar problem respectively in the framework of Hölder spaces and $L^{p}$-spaces. These two last studies have considered only two materials. In our work we will use some techniques of these approachs which are based on the theory of semigroups, the Dunford functional calculus and the interpolation spaces.

This paper is organized as follows.

In Section 1, one gives the formal calculus for the limiting transmission problem and a concrete problem which motivates our study. In section 2, we give the basic hypothesis and some technical lemmas useful to the study of our problem $\left(P_{1 A}\right)$. Section 3 is devoted to the derivation of an explicit representation of the solution of $\left(P_{1 A}\right)$. In Sections 4 and 5 we study the solution and give in addition necessary and sufficient compatibility conditions on the data in order to obtain the above Theorem. In a last Section 6, we go back to the main physical example given in Subsection 1.2 and apply our results in the case of the space $E=C_{0}^{\beta}(\bar{\Omega})$ of the $\beta$-Hölder continuous functions vanishing on the boundary $\partial \Omega$. 


\section{Formal Derivation of the Limiting Effect of the Thin Junction}

\subsection{Derivation of the Transmission Conditions}

In order to have an idea at least formally of the limiting problem, let us first consider the case when operator $-A$ is replaced by a complex scalar $-z$ (with $z \in \mathbb{C} \backslash \mathbb{R}_{+}$) and for the simplicity

$$
\left\{\begin{array}{l}
g_{\mid] 0, \delta[}^{\delta}=0 \\
\alpha^{\delta}=\beta^{\delta}=a^{\delta}=b^{\delta}=0 .
\end{array}\right.
$$

Define functions $w_{+}$and $h_{+}$on the fixed intervall $] 0,1[$ by

$$
w_{+}(x)=u_{+}^{\delta}(\delta+x), \quad h_{+}(x)=g_{+}^{\delta}(\delta+x) .
$$

For simplicity, we have supposed that these functions do not depend on $\delta$.

The equation on the intervall $] 0, \delta[$ writes

$$
\left(u_{0}^{\delta}\right)^{\prime \prime}+z u_{0}^{\delta}=0
$$

which gives

$$
u_{0}^{\delta}(x)=C_{1} e^{-\sqrt{-z} x}+C_{2} e^{-\sqrt{-z}(\delta-x)},
$$

where $C_{1}$ and $C_{2}$ are constants to be fixed by the boundary conditions. We thus have

$$
\left\{\begin{array}{l}
u_{0}^{\delta}(0)=C_{1}+C_{2} e^{-\sqrt{-z} \delta} \\
u_{0}^{\delta}(\delta)=C_{1} e^{-\sqrt{-z} \delta}+C_{2} \\
\left(u_{0}^{\delta}\right)^{\prime}(0)=-C_{1} \sqrt{-z}+C_{2} \sqrt{-z} e^{-\sqrt{-z} \delta} \\
\left(u_{0}^{\delta}\right)^{\prime}(\delta)=-C_{1} \sqrt{-z} e^{-\sqrt{-z} \delta}+\sqrt{-z} C_{2} .
\end{array}\right.
$$

Now, the transmission conditions

$$
\left\{\begin{array}{l}
u_{-}^{\delta}(0)=u_{0}^{\delta}(0) \\
u_{0}^{\delta}(\delta)=u_{+}^{\delta}(\delta)=w_{+}(0) \\
p_{-}\left(u_{-}^{\delta}\right)^{\prime}(0)=p_{0}\left(u_{0}^{\delta}\right)^{\prime}(0) \\
p_{0}\left(u_{0}^{\delta}\right)^{\prime}(\delta)=p_{+}\left(u_{+}^{\delta}\right)^{\prime}(\delta)=p_{+}\left(w_{+}\right)^{\prime}(0)
\end{array}\right.
$$

lead to

$$
2 e^{-\sqrt{-z} \delta} \sqrt{-z} w_{+}(0)=\left(1+e^{-2 \sqrt{-z} \delta}\right) \sqrt{-z} u_{-}^{\delta}(0)+\left(1-e^{-2 \sqrt{-z} \delta}\right) \frac{p_{-}}{p_{0}}\left(u_{-}^{\delta}\right)^{\prime}(0),
$$

and

$$
2 \frac{p_{+}}{p_{0}} e^{-\sqrt{-z} \delta}\left(w_{+}\right)^{\prime}(0)=\left(1-e^{-2 \sqrt{-z} \delta}\right) \sqrt{-z} u_{-}^{\delta}(0)+\frac{p_{-}}{p_{0}}\left(1+e^{-2 \sqrt{-z} \delta}\right)\left(u_{-}^{\delta}\right)^{\prime}(0) .
$$

These two last relations link the Cauchy data $\left(w_{+}(0),\left(w_{+}\right)^{\prime}(0)\right)$ (at the interface $\left.\{0\}\right)$ of the function $w_{+}($defined on $[0,1])$ to the Cauchy data $\left(u_{-}^{\delta}(0),\left(u_{-}^{\delta}\right)^{\prime}(0)\right)$ (at the interface $\{0\})$ of the function $u_{-}^{\delta}$ (defined on $[-1,0]$ ).

The limiting transmission conditions as $\delta \rightarrow 0$, which are obtained from the analysis of (2) and (3) depend on the behavior of $p_{-}, p_{0}$ and $p_{+}$with respect to $\delta$. So we must 
assume some conditions on $p_{-}, p_{0}$ and $p_{+}$. The most interesting case we will consider is the following

$$
p_{-} \text {and } p_{+} \text {are independent of } \delta \text { and } p_{0}=\frac{q}{\delta},
$$

where $q$ is a fixed positive number.

This problem may model an electrostatic potential $u^{\delta}$ in an heterogeneous material (see next subsection). The heterogeneity of the material is translated by the discontinuity of the conductivity coefficient $p$. In this situation the small bond is highly conductive, implying thus the continuity of the potential through the sheet, but the normal component of the electric induction field is no longer continuous through the interface $\{0\}$, it has a jump proportional to the potential at $\{0\}$. In that case one has

$$
\begin{aligned}
& \left(1-e^{-2 \sqrt{-z} \delta}\right) \frac{1}{p_{0}}=\left(1-e^{-2 \sqrt{-z} \delta}\right) \frac{\delta}{q} \rightarrow 0 \quad \text { as } \quad \delta \rightarrow 0, \\
& p_{0}\left(1-e^{-2 \sqrt{-z} \delta}\right)=\frac{q}{\delta}\left(1-e^{-2 \sqrt{-z} \delta}\right) \rightarrow 2 q \sqrt{-z} \quad \text { as } \delta \rightarrow 0,
\end{aligned}
$$

and then the limiting transmission conditions are

$$
\left\{\begin{array}{l}
w_{+}(0)=u_{-}(0) \\
p_{+}\left(w_{+}\right)^{\prime}(0)=q \sqrt{-z} \sqrt{-z} u_{-}(0)+p_{-}\left(u_{-}\right)^{\prime}(0)
\end{array}\right.
$$

and the limiting scalar problem becomes

$$
\left(P_{1 z}\right)\left\{\begin{array}{l}
\left.\left(u_{-}\right)^{\prime \prime}(x)+z u_{-}(x)=g_{-}(x) \text { on }\right]-1,0[ \\
\left.\left(w_{+}\right)^{\prime \prime}(x)+z w_{+}(x)=h_{+}(x) \text { on }\right] 0,1[ \\
u(-1)=f_{-}, \quad\left(w_{+}\right)^{\prime}(1)=f_{+} \\
u_{-}(0)=w_{+}(0) \\
p_{-}\left(u_{-}\right)^{\prime}(0)-p_{+}\left(w_{+}\right)^{\prime}(0)=q z u_{-}(0)
\end{array}\right.
$$

Remark 1. Another interesting case is when $p_{-}$and $p_{+}$are constant with respect to $\delta$ but $p_{0}=\delta q$. In that case the small bond is not sufficiently conductive, implying thus a jump of the potential at $\{0\}$, but the normal component of the electric induction field remains continuous through the interface $\{0\}$ and it is proportional to the jump of the potential at $\{0\}$. In that case, one has

$$
\begin{aligned}
& \left(1-e^{-2 \sqrt{-z} \delta}\right) \frac{1}{p_{0}}=\left(1-e^{-2 \sqrt{-z} \delta}\right) \frac{1}{q \delta} \rightarrow 2 \frac{1}{q} \sqrt{-z} \text { a s } \delta \rightarrow 0 \\
& p_{0}\left(1-e^{-2 \sqrt{-z} \delta}\right)=q \delta\left(1-e^{-2 \sqrt{-z} \delta}\right) \rightarrow 0 \text { as } \delta \rightarrow 0
\end{aligned}
$$

and then the transmission conditions are

$$
\left\{\begin{array}{l}
q w_{+}(0)=q u_{-}(0)+p_{+}\left(u_{-}\right)^{\prime}(0) \\
p_{+}\left(w_{+}\right)^{\prime}(0)=p_{-}\left(u_{-}\right)^{\prime}(0)
\end{array}\right.
$$

The limiting problem becomes

$$
\left(P_{2 A}\right)\left\{\begin{array}{l}
\left.\left(u_{-}\right)^{\prime \prime}(x)+A u_{-}(x)=g_{-}(x) \text { on }\right]-1,0[ \\
\left.\left(w_{+}\right)^{\prime \prime}(x)+A w_{+}(x)=h_{+}(x) \text { on }\right] 0,1[ \\
u(-1)=f_{-},\left(w_{+}\right)^{\prime}(1)=f_{+}, \\
\left.p_{-}\left(u_{-}\right)^{\prime}(0)=q\left(u_{-}(0)-w_{+}(0)\right)=p_{+}\left(w_{+}\right)^{\prime}(0)\right) .
\end{array}\right.
$$


In the general case where the data $g_{\mid] 0, \delta[}^{\delta}, \alpha^{\delta}, \beta^{\delta}, a^{\delta}$ and $b^{\delta}$ are different from 0 , one obtains the following relations between $\left(w_{+}(0),\left(w_{+}\right)^{\prime}(0)\right)$ and $\left(u_{-}^{\delta}(0),\left(u_{-}^{\delta}\right)^{\prime}(0)\right)$

$$
\begin{aligned}
& 2 e^{-\sqrt{-z} \delta} \sqrt{-z} w_{+}(0)= \\
& =\left(1+e^{-2 \sqrt{-z} \delta}\right) \sqrt{-z} u_{-}^{\delta}(0)+\left(1-e^{-2 \sqrt{-z} \delta}\right) \frac{p_{-}}{p_{0}}\left(u_{-}^{\delta}\right)^{\prime}(0)+\varphi^{\delta}\left(g_{\mid] 0, \delta[}^{\delta}, \alpha^{\delta}, \beta^{\delta}, a^{\delta}\right)
\end{aligned}
$$

and

$$
\begin{aligned}
& 2 e^{-\sqrt{-z} \delta}\left(w_{+}\right)^{\prime}(0) \frac{p_{+}}{p_{0}}= \\
& =\left(1-e^{-2 \sqrt{-z} \delta}\right) \sqrt{-z} u_{-}^{\delta}(0)+\left(1+e^{-2 \sqrt{-z} \delta}\right)\left(u_{-}^{\delta}\right)^{\prime}(0) \frac{p_{-}}{p_{0}}+\psi^{\delta}\left(g_{\mid] 0, \delta[}^{\delta}, \alpha^{\delta}, a^{\delta}, b^{\delta}\right)
\end{aligned}
$$

where

$$
\begin{aligned}
& \varphi^{\delta}\left(g_{\mid] 0, \delta[}^{\delta}, \alpha^{\delta}, \beta^{\delta}, a^{\delta}\right)= \\
& =-\left(1-e^{-2 \sqrt{-z} \delta}\right) \int_{0}^{\delta} e^{-\sqrt{-z} s} g_{0}^{\delta}(s) d s-\left(1-e^{-2 \sqrt{-z} \delta}\right)\left(\sqrt{-z} \alpha^{\delta}+\frac{a^{\delta}}{p_{0}}\right)-2 \sqrt{-z} e^{-\sqrt{-z} \delta} \beta^{\delta}
\end{aligned}
$$

and

$$
\begin{aligned}
& \psi^{\delta}\left(g_{\mid] 0, \delta[}^{\delta}, \alpha^{\delta}, a^{\delta}, b^{\delta}\right)= \\
& =\left(1-e^{-2 \sqrt{-z} \delta}\right) \int_{0}^{\delta} e^{-\sqrt{-z} s} g_{0}^{\delta}(s) d s+\left(1-e^{-2 \sqrt{-z} \delta}\right)\left(\sqrt{-z} \alpha^{\delta}+\frac{a^{\delta}}{p_{0}}\right)-\frac{b^{\delta}}{p_{0}}
\end{aligned}
$$

When we assume (4), we obtain the following limiting scalar problem with non homogeneous transmission conditions

$$
\left(P_{1 z}\right)\left\{\begin{array}{l}
\left.\left(u_{-}\right)^{\prime \prime}(x)+z u_{-}(x)=g_{-}(x) \text { on }\right]-1,0[ \\
\left.\left(w_{+}\right)^{\prime \prime}(x)+z w_{+}(x)=h_{+}(x) \text { on }\right] 0,1[ \\
u(-1)=f_{-}, \quad\left(w_{+}\right)^{\prime}(1)=f_{+} \\
u_{-}(0)=w_{+}(0)+\varphi \\
p_{-}\left(u_{-}\right)^{\prime}(0)-p_{+}\left(w_{+}\right)^{\prime}(0)=q z u_{-}(0)+\psi
\end{array}\right.
$$

where

$$
\varphi=\lim _{\delta \rightarrow 0} \varphi^{\delta}\left(g_{\mid] 0, \delta[}^{\delta}, \alpha^{\delta}, \beta^{\delta}, a^{\delta}\right), \quad \psi=\lim _{\delta \rightarrow 0} \psi^{\delta}\left(g_{\mid] 0, \delta[}^{\delta}, \alpha^{\delta}, a^{\delta}, b^{\delta},\right) .
$$

Therefore, in this work we will focus ourselves on the complete analysis of the following problem

$$
\left(P_{1 A}\right)\left\{\begin{array}{l}
\left.\left(u_{-}\right)^{\prime \prime}(x)+A u_{-}(x)=g_{-}(x) \text { on }\right]-1,0[ \\
\left.\left(w_{+}\right)^{\prime \prime}(x)+A w_{+}(x)=h_{+}(x) \text { on }\right] 0,1[ \\
u(-1)=f_{-},\left(w_{+}\right)^{\prime}(1)=f_{+} \\
u_{-}(0)=w_{+}(0)+\varphi \\
p_{-}\left(u_{-}\right)^{\prime}(0)-p_{+}\left(w_{+}\right)^{\prime}(0)=q A u_{-}(0)+\psi
\end{array}\right.
$$

\subsection{Electrostatic Potential in a Heterogeneous Cylinder}

Consider the cylinder $\left.G^{\delta}=\right]-1,1+\delta[\times \Omega$ constituted by the junction of two homogeneous cylinders $\left.G_{-}=\right]-1,0\left[\times \Omega\right.$ and $\left.G_{+}^{\delta}=\right] \delta, 1+\delta[\times \Omega$ bonded together by the thin cylinder $\left.G_{0}^{\delta}=\right] 0, \delta\left[\times \Omega\right.$ (here $\Omega$ is a bounded domain of $\mathbb{R}^{n}, n \geqslant 1$, with a regular boundary $\Gamma)$. Denote by $(x, y)$ the generic variable in $G^{\delta}$. 
The transmission problem

$$
\left\{\begin{array}{l}
\nabla \cdot\left(p \nabla u^{\delta}\right)=p g^{\delta} \text { in } G^{\delta} \\
\left.u^{\delta}=0 \text { on }\right]-1,1+\delta[\times \Gamma \\
u^{\delta}=f_{-} \text {on }\{-1\} \times \Omega \\
p \frac{\partial u^{\delta}}{\partial x}=f_{+} \text {on }\{1+\delta\} \times \Omega
\end{array}\right.
$$

models an electrostatic problem in $G^{\delta}$. The function $u^{\delta}$ is the electrostatic potential, $-\nabla u^{\delta}$ is the electric field and $-p \nabla u^{\delta}$ is the electric induction field. The heterogeneity of the material is translated by the discontinuity of the conductivity coefficient $p$ :

$$
p=\left\{\begin{array}{l}
\left.p_{-} \text {in }\right]-1,0[\times \Omega \\
\left.p_{0} \text { in }\right] 0, \delta[\times \Omega \\
\left.p_{+} \text {in }\right] \delta, 1+\delta[\times \Omega .
\end{array}\right.
$$

where $p_{-}, p_{0}$ and $p_{+}$are positive constants. The function $g$ is a given electric density, $f_{-}$ is a fixed surface potential and $f_{+}$a fixed surface induction.

Set

then, the equation

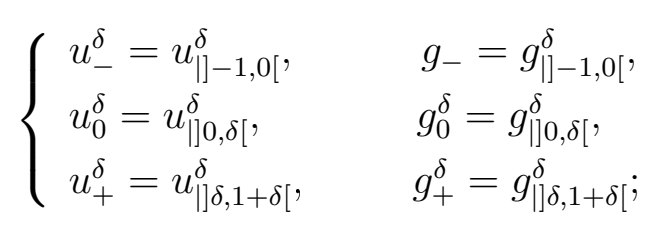

$$
\nabla \cdot\left(p \nabla u^{\delta}\right)=p g^{\delta} \text { in } G^{\delta}
$$

is equivalent to the following equations

$$
\left\{\begin{array}{l}
\left.\Delta u_{-}^{\delta}=g_{-} \text {in }\right]-1,0[\times \Omega \\
\left.\Delta u_{0}^{\delta}=g_{0}^{\delta} \text { in }\right] 0, \delta[\times \Omega \\
\left.\Delta u_{+}^{\delta}=g_{+}^{\delta} \text { in }\right] \delta, 1+\delta[\times \Omega,
\end{array}\right.
$$

with the transmission conditions

$$
\left\{\begin{array}{l}
u_{-}^{\delta}=u_{0}^{\delta} \text { on }\{0\} \times \Omega \\
u_{0}^{\delta}=u_{+}^{\delta} \text { on }\{\delta\} \times \Omega \\
p_{-} \frac{\partial u_{-}^{\delta}}{\partial x}=p_{0} \frac{\partial u_{0}^{\delta}}{\partial x} \text { on }\{0\} \times \Omega \\
p_{0} \frac{\partial u_{0}^{\delta}}{\partial x}=p_{+} \frac{\partial u_{+}^{\delta}}{\partial x} \text { on }\{\delta\} \times \Omega .
\end{array}\right.
$$

Let us define the functions $w_{+}, h_{+}$on the fixed domain $] 0,1[\times \Omega$ by setting

$$
w_{+}(x, y)=u_{+}^{\delta}(\delta+x, y), \quad h_{+}(x, y)=g_{+}^{\delta}(\delta+x, y)
$$

where we have assumed, for simplicity, that they do not depend on $\delta$.

The approximations for $u_{0}^{\delta}(x,$.$) and \frac{\partial u_{0}^{\delta}}{\partial x}(x,$.$) as \delta \rightarrow 0$, give

$$
\begin{aligned}
u_{0}^{\delta}(\delta, y) & \simeq u_{0}^{\delta}(0, y)+\delta \frac{\partial u_{0}^{\delta}}{\partial x}(0, y)+\frac{\delta^{2}}{2} \frac{\partial^{2} u_{0}^{\delta}}{\partial x^{2}}(0, y)= \\
& =u_{0}^{\delta}(0, y)+\delta \frac{\partial u_{0}^{\delta}}{\partial x}(0, y)-\frac{\delta^{2}}{2}\left(\Delta_{y} u_{0}^{\delta}+g_{0}\right)(0, y)
\end{aligned}
$$




$$
\frac{\partial u_{0}^{\delta}}{\partial x}(\delta, y) \simeq \frac{\partial u_{0}^{\delta}}{\partial x}(0, y)+\delta \frac{\partial^{2} u_{0}^{\delta}}{\partial x^{2}}(0, y)=\frac{\partial u_{0}^{\delta}}{\partial x}(0, y)-\delta\left(\Delta_{y} u_{0}^{\delta}-g_{0}\right)(0, y)
$$

Using these relations with (11) and (12), we get the following problem set on the fixed domain (not depending on $\delta$ ):

$$
(E Q S)\left\{\begin{array}{l}
\left.\Delta u_{-}=g_{-} \text {in }\right]-1,0[\times \Omega \\
\left.\Delta w_{+}=h_{+} \text {in }\right] 0,1[\times \Omega
\end{array}\right.
$$

under the boundary conditions

$$
(B C)\left\{\begin{array}{l}
\left.u_{-}=0 \text { on }\right]-1,0[\times \Gamma \\
\left.w_{+}=0 \text { on }\right] 0,1[\times \Gamma \\
u_{-}=f_{-} \text {on }\{-1\} \times \Omega \\
p_{+} \frac{\partial w_{+}}{\partial x}=f_{+} \text {on }\{1\} \times \Omega
\end{array}\right.
$$

and the following transmission conditions (depending on $\delta$ )

$$
(T C)\left\{\begin{array}{c}
w_{+}=u_{-}+\delta \frac{p_{-}}{p_{0}} \frac{\partial u_{-}}{\partial x}-\frac{\delta^{2}}{2}\left(\Delta_{y} u_{0}-g_{0}\right) \text { on }\{0\} \times \Omega \\
p_{+} \frac{\partial w_{+}}{\partial x}=p_{-} \frac{\partial u_{-}}{\partial x}-\delta p_{0}\left(\Delta_{y} u_{-}-g_{0}\right) \text { on }\{0\} \times \Omega,
\end{array}\right.
$$

which model the effect of the thin cylinder $] 0, \delta[\times \Omega$ on the other parts of the cylinder. The thin cylinder $] 0, \delta[\times \Omega$ is modelled by the sheet $\{0\} \times \Omega$ and its effect is modelled through these transmission conditions.

There are two limiting cases of particular interest. The first case is

$$
p_{0}=\frac{q}{\delta}
$$

( $q$ is a positive constant) which is considered in this work assumes that the thin layer is highly conductive; as $\delta$ goes to 0 , we obtain

$$
\left\{\begin{array}{l}
w_{+}=u_{-} \text {on }\{0\} \times \Omega \\
p_{+} \frac{\partial w_{+}}{\partial x}=p_{-} \frac{\partial u_{-}}{\partial x}-q\left(\Delta_{y} u_{-}+g_{0}\right) \text { on }\{0\} \times \Omega,
\end{array}\right.
$$

which corresponds to the fact that the potential is continuous through the sheet, but the normal component of the electric induction field has a jump proportional to the potential.

The second case is $p_{0}=q \delta$ and corresponds to the fact that the thin layer is poorly conductive. We get, as $\delta$ goes to 0 :

$$
\left\{\begin{array}{l}
w_{+}=u_{-}+\frac{1}{q} p_{-} \frac{\partial u_{-}}{\partial x} \text { on }\{0\} \times \Omega, \\
p_{+} \frac{\partial w_{+}}{\partial x}=p_{-} \frac{\partial u_{-}}{\partial x} \text { on }\{0\} \times \Omega
\end{array}\right.
$$

here, the normal component of the electric induction is continuous through the sheet but the potential has a jump proportional to the normal componant of the electric induction field. 
Therefore, using the classical operational notations

$$
u_{-}(x)(y):=u_{-}(x, y), \quad w_{+}(x)(y):=w_{+}(x, y), \ldots
$$

the concrete problem (13), (14), (15) writes exactly in the form (9) with

$$
\psi=q g_{0} \text { and } \varphi=0
$$

and

$$
\left\{\begin{array}{l}
D(A)=\left\{v \in W^{2, p}(0,1): v(0)=v(1)=0\right\} \\
A v(y)=v^{\prime \prime}(y)
\end{array}\right.
$$

in the case $E=L^{p}(0,1)$, or

$$
\left\{\begin{array}{l}
D(A)=\left\{v \in C^{2}[0,1]: v(0)=v(1)=0\right\} \\
A v(y)=v^{\prime \prime}(y)
\end{array}\right.
$$

in the case $E=C[0,1]$.

\section{Hypotheses and Technical Lemmas}

We assume in all this work the following ellipticity hypothesis:

$$
\left\{\begin{array}{l}
\text { for any } \theta \in] 0, \pi\left[\rho(A) \supset S_{\pi-\theta} \cup\{0\}\right. \text { and } \\
\exists C>0: \forall \lambda \in S_{\pi-\theta} \cup\{0\} \quad\left\|(\lambda I-A)^{-1}\right\|_{L(X)} \leqslant \frac{C}{1+|\lambda|},
\end{array}\right.
$$

where $\varrho(A)$ denotes the resolvent set of $A$ and

$$
S_{\theta}=\{z \in \mathbb{C} \backslash\{0\}:|\arg z|<\theta\} .
$$

This assumption implies that there exist a ball $B\left(0, r_{0}\right), r_{0}>0$, such that

$$
\varrho(A) \supset \overline{B\left(0, r_{0}\right)},
$$

and the estimate in (16) is still true in $S_{\pi-\theta} \cup \overline{B\left(0, r_{0}\right)}$.

It is well known that the above assumption implies that the square root $\sqrt{-A}$ is well defined and $B=-\sqrt{-A}$ generates an analytic semigroup

$$
\left(e^{B \xi}\right)_{\xi>0}
$$

which is not necessarily continuous at 0 .

Let us recall the following important properties of $B$ proved in [7].

Lemma 1. Let $\phi \in E$ and $\chi \in C^{\eta}([0, T] ; E)$ with $T>0$. Then

1. $e^{B \xi} \phi \rightarrow \phi$ as $\xi \rightarrow 0^{+}$iff $\phi \in \overline{D(B)}=\overline{D(A)}$, (see [7, p. 20, Proposition 1.2]),

2. $\xi \mapsto e^{B \xi} \phi \in C^{\eta}([0, T], E)$ iff $\phi \in D_{B}(\eta ;+\infty)=D_{A}(\eta / 2 ;+\infty)$, (see [7, p. 29, Proposition 1.12]), 
3. $\xi \mapsto \int_{0}^{\xi} B e^{(\xi-t) B}[\chi(t)-\chi(\xi)] d t \in C^{\eta}([0, T], E) \cap B\left(0, T ; D_{B}(\eta ;+\infty)\right)$, (see $[$ \% , p. 53, Theorem 4.5]).

Let $\theta \in] 0, \pi / 2[$ and set

$$
S\left(r_{0}, \theta\right)=\left\{z \in \mathbb{C} \backslash\{0\}:|z| \geqslant r_{0} \sin \theta \text { and }|\arg z|<\theta\right\} .
$$

Note that for all $w \in S\left(r_{0}, \theta\right)$, one clearly has

$$
\text { Re } w \geqslant r_{0} \sin \theta
$$

We will also use the following result proved in [6, p. 1880, Proposition 4.10].

Lemma 2. For any $w \in S\left(r_{0}, \theta\right)$, one has

1. $\left|\arg \left(1-e^{-w}\right)-\arg \left(1+e^{-w}\right)\right|<\theta$,

2. $\left|1+e^{-w}\right| \geqslant C_{\theta}=1-e^{-\frac{\pi}{2 \tan \theta}}>0$,

3. $\left|1-e^{-w}\right| \geq \frac{\operatorname{Re} w}{1+\operatorname{Re} w} \geqslant \frac{r_{0} \sin \theta}{1+r_{0} \sin \theta}=c_{1}$.

Now, consider the following space

$$
H^{\infty}\left(S\left(r_{0}, \theta\right)\right)=\left\{f: f \text { is an holomorphic and bounded function on } S\left(r_{0}, \theta\right)\right\},
$$

then, under our assumptions on $A$, if $f \in H^{\infty}\left(S\left(r_{0}, \theta\right)\right)$ is such that $1 / f \in H^{\infty}\left(S\left(r_{0}, \theta\right)\right)$ and $(1 / f)(-B) \in \mathcal{L}(X)$, then $f(-B)$ is invertible with a bounded inverse and

$$
[f(-B)]^{-1}=(1 / f)(-B)
$$

see, for instance [8] or [9, p. 45, Remark 2.5.1].

On the other hand we recall that operator $I-e^{2 B}$ and $I+e^{2 B}$ are boundedly invertible; see for instance [10, p. 60, Proposition 2.3.6].

Let us now apply this result to the following operator

$$
\Delta_{*}=I-\frac{p_{-}}{q} B^{-1}\left(I-e^{2 B}\right)^{-1}\left(I+e^{2 B}\right)-\frac{p_{+}}{q} B^{-1}\left(I+e^{2 B}\right)^{-1}\left(I-e^{2 B}\right) .
$$

Lemma 3. The operator $\Delta_{*}: E \rightarrow E$ is boundedly invertible.

Proof. Let $0<\theta<\pi / 2$. Consider the function $f$

$$
f(w)=1+\frac{p_{-}}{q w} \frac{1+e^{-2 w}}{1-e^{-2 w}}+\frac{p_{+}}{q w} \frac{1-e^{-2 w}}{1+e^{-2 w}} .
$$

It is clear that $f$ and $1 / f$ are well defined and holomorphic on $S\left(r_{0}, \theta\right)$ in virtue of the above Lemma. The function $f$ is clearly bounded on $S\left(r_{0}, \theta\right)$. Moreover there exists $C>0$ such that for all $w \in S\left(r_{0}, \theta\right)$ one has 


$$
\left|\frac{p_{-}}{q w} \frac{1+e^{-2 w}}{1-e^{-2 w}}+\frac{p_{+}}{q w} \frac{1-e^{-2 w}}{1+e^{-2 w}}\right| \leq \frac{C}{|w|} .
$$

Therefore one can find $x_{\theta}>0$ such that

$$
\left|\frac{p_{-}}{q w} \frac{1+e^{-2 w}}{1-e^{-2 w}}+\frac{p_{+}}{q w} \frac{1-e^{-2 w}}{1+e^{-2 w}}\right| \leq \frac{1}{2}
$$

for $w \in S\left(r_{0}, \theta\right)$ with Re $w \geq x_{\theta}$; consequently

$$
\begin{aligned}
|f(w)| & =\left|1+\frac{p_{-}}{q w} \frac{1+e^{-2 w}}{1-e^{-2 w}}+\frac{p_{+}}{q w} \frac{1-e^{-2 w}}{1+e^{-2 w}}\right| \\
& \geq 1-\left|\frac{p_{-}}{q w} \frac{1+e^{-2 w}}{1-e^{-2 w}}+\frac{p_{+}}{q w} \frac{1-e^{-2 w}}{1+e^{-2 w}}\right| \\
& \geq 1 / 2
\end{aligned}
$$

for $w \in S\left(r_{0}, \theta\right)$ with $R e w \geq x_{\theta}$.

In the compact sector

$$
K_{x_{\theta}}=\left\{w \in \mathbb{C} \backslash\{0\}: r_{0} \sin \theta \leqslant \operatorname{Re} w \leqslant x_{\theta} \text { and }|\arg (w)| \leqslant \theta\right\},
$$

there is at most a finite number of roots of $f(w)$ (not belonging to $\left[0, x_{\theta}\right]$ ), (see [11, the remark on Proposition 4.1 , p. 41]); so there exists $\left.\left.\theta^{*} \in\right] 0, \theta\right]$ such that $f(w)$ does not vanish on

$$
\Sigma_{r_{0}, \theta^{*}}:=\left\{w \in \mathbb{C} \backslash\{0\}: r_{0} \sin \theta \leqslant \operatorname{Re} w \leqslant x_{\theta} \text { and }|\arg (w)| \leqslant \theta^{*}\right\} .
$$

From (21), we conclude that $f(w)$ does not vanish on $\overline{S\left(r_{0}, \theta^{*}\right)}$. Hence $f \in H^{\infty}\left(S\left(r_{0}, \theta^{*}\right)\right)$, $1 / f \in H^{\infty}\left(S\left(r_{0}, \theta^{*}\right)\right)$ and consequently $(1 / f)(-B) \in \mathcal{L}(X)$ and

$$
\Delta_{*}=f(-B)=I-\frac{p_{-}}{q} B^{-1}\left(I-e^{2 B}\right)^{-1}\left(I+e^{2 B}\right)-\frac{p_{+}}{q} B^{-1}\left(I+e^{2 B}\right)^{-1}\left(I-e^{2 B}\right)
$$

is boundedly invertible.

\section{Representation of the Solution of $\left(P_{1 A}\right)$}

It is well known that, the solution of the second order following equation

$$
\left.u^{\prime \prime}(x)-B^{2} u(x)=g(x), x \in\right] a, b[
$$

in $E$, writes

$$
u(x)=e^{(x-a) B} \alpha+e^{(b-x) B} \beta+v(g)(x)
$$

with $\alpha, \beta \in E$ and

$$
v(g)(x)=\frac{1}{2} \int_{a}^{x} e^{(x-t) B} B^{-1} g(t) d t+\frac{1}{2} \int_{x}^{b} e^{(t-x) B} B^{-1} g(t) d t .
$$


Therefore

$$
\begin{array}{ll}
u_{-}(x)=e^{-x B} \alpha_{-}+e^{(1+x) B} \beta_{-}+v\left(g_{-}\right)(x), & x \in]-1,0[ \\
w_{+}(x)=e^{x B} \alpha_{+}+e^{(1-x) B} \beta_{+}+v\left(h_{+}\right)(x), & x \in] 0,1[
\end{array}
$$

with $\alpha_{-}, \beta_{-}, \alpha_{+}, \beta_{+} \in E$ and

$$
\begin{aligned}
& v\left(g_{-}\right)(x)=\frac{1}{2} \int_{-1}^{x} e^{(x-t) B} B^{-1} g_{-}(t) d t+\frac{1}{2} \int_{x}^{0} e^{(t-x) B} B^{-1} g_{-}(t) d t, \\
& v\left(h_{+}\right)(x)=\frac{1}{2} \int_{0}^{x} e^{(x-t) B} B^{-1} h_{+}(t) d t+\frac{1}{2} \int_{x}^{1} e^{(t-x) B} B^{-1} h_{+}(t) d t .
\end{aligned}
$$

The boundary conditions

$$
\left\{\begin{array}{l}
u_{-}(-1)=e^{B} \alpha_{-}+\beta_{-}+v\left(g_{-}\right)(-1)=f_{-} \\
w_{+}^{\prime}(1)=B e^{B} \alpha_{+}-B \beta_{+}+v^{\prime}\left(h_{+}\right)(1)=f_{+},
\end{array}\right.
$$

give

$$
\left\{\begin{array}{l}
\beta_{-}=-e^{B} \alpha_{-}-v\left(g_{-}\right)(-1)+f_{-} \\
\beta_{+}=e^{B} \alpha_{+}+B^{-1} v^{\prime}\left(h_{+}\right)(1)-B^{-1} f_{+}
\end{array}\right.
$$

and the transmission conditions

$$
\left\{\begin{array}{l}
u_{-}(0)=w_{+}(0)+\varphi \\
p_{-}\left(u_{-}\right)^{\prime}(0)-p_{+}\left(w_{+}\right)^{\prime}(0)=q A u_{-}(0)+\psi
\end{array}\right.
$$

give

$$
\left\{\begin{array}{l}
\alpha_{-}+e^{B} \beta_{-}-\alpha_{+}-e^{B} \beta_{+} \\
=\varphi-v\left(g_{-}\right)(0)+v\left(h_{+}\right)(0) \\
p_{-}\left(-\alpha_{-}+e^{B} \beta_{-}\right)-p_{+}\left(\alpha_{+}-e^{B} \beta_{+}\right)+q B\left(\alpha_{-}+e^{B} \beta_{-}\right) \\
=-p_{-} B^{-1} v^{\prime}\left(g_{-}\right)(0)+p_{+} B^{-1} v^{\prime}\left(h_{+}\right)(0)-q B v\left(g_{-}\right)(0)+B^{-1} \psi .
\end{array}\right.
$$

Using (23), one obtains the system

$$
\left\{\begin{array}{l}
\left(I-e^{2 B}\right) \alpha_{-}-\left(I+e^{2 B}\right) \alpha_{+}=F_{*} \\
{\left[-p_{-}\left(I+e^{2 B}\right)+q B\left(I-e^{2 B}\right)\right] \alpha_{-}-p_{+}\left(I-e^{2 B}\right) \alpha_{+}=G_{*},}
\end{array}\right.
$$

where

$$
\left\{\begin{array}{l}
F_{*}=\varphi-e^{B} f_{-}-e^{B} B^{-1} f_{+}+e^{B} v\left(g_{-}\right)(-1) \\
-v\left(g_{-}\right)(0)+e^{B} B^{-1} v^{\prime}\left(h_{+}\right)(1)+v\left(h_{+}\right)(0) \\
G_{*}=B^{-1} \psi-\left(p_{-}+q B\right) e^{B} f_{-}+p_{+} e^{B} B^{-1} f_{+} \\
+\left(p_{-}+q B\right) e^{B} v\left(g_{-}\right)(-1)-p_{+} e^{B} B^{-1} v^{\prime}\left(h_{+}\right)(1) \\
-p_{-} B^{-1} v^{\prime}\left(g_{-}\right)(0)+p_{+} B^{-1} v^{\prime}\left(h_{+}\right)(0)-q B v\left(g_{-}\right)(0) .
\end{array}\right.
$$

The abstract determinant of this system (acting on $D(B)$ ) is

$$
\begin{aligned}
& \Delta(B)= \\
& =p_{+}\left(I-e^{2 B}\right)\left(I-e^{2 B}\right)-\left(I+e^{2 B}\right)\left[-p_{-}\left(I+e^{2 B}\right)+q B\left(I-e^{2 B}\right)\right]= \\
& =p_{+}\left(I-e^{2 B}\right)^{2}+p_{-}\left(I+e^{2 B}\right)^{2}-q B\left(I-e^{2 B}\right)\left(I+e^{2 B}\right) .
\end{aligned}
$$


One has

$$
\Delta(B): D(B) \rightarrow E
$$

with

$$
\begin{aligned}
& -\Delta(B)=-p_{+}\left(I-e^{2 B}\right)^{2}-p_{-}\left(I+e^{2 B}\right)^{2}+q B\left(I-e^{2 B}\right)\left(I+e^{2 B}\right) \\
& =q B\left(I-e^{2 B}\right)\left(I+e^{2 B}\right) \\
& \quad\left[I-\frac{p_{-}}{q} B^{-1}\left(I-e^{2 B}\right)^{-1}\left(I+e^{2 B}\right)-\frac{p_{+}}{q} B^{-1}\left(I+e^{2 B}\right)^{-1}\left(I-e^{2 B}\right)\right] \\
& \quad:=q B\left(I-e^{2 B}\right)\left(I+e^{2 B}\right) \Delta_{*},
\end{aligned}
$$

which is boundedly invertible by Lemma 4 . It follows that

$$
\begin{gathered}
\alpha_{-}=[\Delta(B)]^{-1}\left[p_{+}\left(I-e^{2 B}\right) F_{*}-\left(I+e^{2 B}\right) G_{*}\right] \\
\alpha_{+}=[\Delta(B)]^{-1}\left[\left(-p_{-}\left(I+e^{2 B}\right)+q B\left(I-e^{2 B}\right)\right) F_{*}-\left(I-e^{2 B}\right) G_{*}\right],
\end{gathered}
$$

and then

$$
\begin{aligned}
& \beta_{-}=-e^{B}[\Delta(B)]^{-1}\left[p_{+}\left(I-e^{2 B}\right) F_{*}-\left(I+e^{2 B}\right) G_{*}\right]-v\left(g_{-}\right)(-1)+f_{-}, \\
& \beta_{+}=e^{B}[\Delta(B)]^{-1}\left[\left(-p_{-}\left(I+e^{2 B}\right)+q B\left(I-e^{2 B}\right)\right) F_{*}-\left(I-e^{2 B}\right) G_{*}\right] \\
& +B^{-1} v^{\prime}\left(h_{+}\right)(1)-B^{-1} f_{+} .
\end{aligned}
$$

Therefore, the functions

$$
\begin{cases}u_{-}(x)=e^{-x B} \alpha_{-}+e^{(1+x) B} \beta_{-}+v\left(g_{-}\right)(x), & x \in]-1,0[ \\ w_{+}(x)=e^{x B} \alpha_{+}+e^{(1-x) B} \beta_{+}+v\left(h_{+}\right)(x), & x \in] 0,1[\end{cases}
$$

with

$$
v\left(g_{-}\right)(x)=\frac{1}{2} \int_{-1}^{x} e^{(x-t) B} B^{-1} g_{-}(t) d t+\frac{1}{2} \int_{x}^{0} e^{(t-x) B} B^{-1} g_{-}(t) d t
$$

and

$$
v\left(h_{+}\right)(x)=\frac{1}{2} \int_{0}^{x} e^{(x-t) B} B^{-1} h_{+}(t) d t+\frac{1}{2} \int_{x}^{1} e^{(t-x) B} B^{-1} h_{+}(t) d t
$$

are completely determined. 


\section{Analysis of $v\left(g_{-}\right)$and $v\left(h_{+}\right)$and Their Derivatives}

\subsection{Analysis of $v\left(g_{-}\right)$on $(-1,0)$}

One has

$$
\begin{aligned}
& v\left(g_{-}\right)(x) \\
& =\frac{1}{2} \int_{-1}^{x} e^{(x-t) B} B^{-1}\left[g_{-}(t)-g_{-}(x)\right] d t+\frac{1}{2} \int_{x}^{0} e^{(t-x) B} B^{-1}\left[g_{-}(t)-g_{-}(x)\right] d t \\
& +\frac{1}{2} \int_{-1}^{x} e^{(x-t) B} B^{-1} g_{-}(x) d t+\frac{1}{2} \int_{x}^{0} e^{(t-x) B} B^{-1} g_{-}(x) d t \\
& =\frac{1}{2} \int_{-1}^{x} e^{(x-t) B} B^{-1}\left[g_{-}(t)-g_{-}(x)\right] d t+\frac{1}{2} \int_{x}^{0} e^{(t-x) B} B^{-1}\left[g_{-}(t)-g_{-}(x)\right] d t \\
& +\frac{1}{2} e^{(x+1) B} B^{-2} g_{-}(x)+\frac{1}{2} e^{-x B} B^{-2} g_{-}(x)-B^{-2} g_{-}(x),
\end{aligned}
$$

and

$$
\begin{aligned}
& v\left(g_{-}\right)(x) \\
& =\frac{1}{2} \int_{-1}^{x} e^{(x-t) B} B^{-1}\left[g_{-}(t)-g_{-}(x)\right] d t+\frac{1}{2} \int_{x}^{0} e^{(t-x) B} B^{-1}\left[g_{-}(t)-g_{-}(x)\right] d t \\
& +\frac{1}{2} e^{(x+1) B} B^{-2}\left[g_{-}(x)-g_{-}(-1)\right]+\frac{1}{2} e^{-x B} B^{-2}\left[g_{-}(x)-g_{-}(0)\right]-B^{-2} g_{-}(x) \\
& +\frac{1}{2} e^{(x+1) B} B^{-2} g_{-}(-1)+\frac{1}{2} e^{-x B} B^{-2} g_{-}(0)=v_{R}\left(g_{-}\right)(x)+v_{S,-1}\left(g_{-}\right)(x)+v_{S, 0}\left(g_{-}\right)(x),
\end{aligned}
$$

where

$$
v_{S,-1}\left(g_{-}\right)(x)=\frac{1}{2} e^{(x+1) B} B^{-2} g_{-}(-1) ; \quad v_{S, 0}\left(g_{-}\right)(x)=\frac{1}{2} e^{-x B} B^{-2} g_{-}(0) .
$$

Due to Lemma $1, v_{R}\left(g_{-}\right)$has the following maximal regularity properties

$$
\left\{\begin{array}{c}
v_{R}\left(g_{-}\right) \in C\left([-1,0] ; D\left(B^{2}\right)\right) \\
B^{2} v_{R}\left(g_{-}\right) \in C^{\eta}([-1,0] ; E)
\end{array}\right.
$$

while for $v_{S,-1}\left(g_{-}\right)$and $v_{S, 0}\left(g_{-}\right)(x)$ we only have

$$
\left\{\begin{array}{l}
\left.\left.\left.\left.v_{S,-1}\left(g_{-}\right) \in C(]-1,0\right] ; D\left(B^{2}\right)\right) ; \quad B^{2} v_{S,-1}\left(g_{-}\right) \in C^{\eta}(]-1,0\right] ; E\right) \\
v_{S, 0}\left(g_{-}\right) \in C\left(\left[-1,0\left[; D\left(B^{2}\right)\right) ; \quad B^{2} v_{S, 0}\left(g_{-}\right) \in C^{\eta}([-1,0[; E) .\right.\right.
\end{array}\right.
$$

The behaviour of $B^{2} v_{S,-1}\left(g_{-}\right)(x)$ in the neighbourhood of -1 is that of

$$
\frac{1}{2} e^{(x+1) B} g_{-}(-1)
$$

and the behaviour of $B^{2} v_{S, 0}\left(g_{-}\right)(x)$ in the neighbourhood of 0 is that of

$$
\frac{1}{2} e^{(x+1) B} g_{-}(0) \text {. }
$$


4.2. Analysis of $v\left(h_{+}\right)$on $(0,1)$

Recall that

$$
v\left(h_{+}\right)(x)=\frac{1}{2} \int_{0}^{x} e^{(x-t) B} B^{-1} h_{+}(t) d t+\frac{1}{2} \int_{x}^{1} e^{(t-x) B} B^{-1} h_{+}(t) d t,
$$

which can be written as

$$
\begin{aligned}
& v\left(h_{+}\right)(x) \\
& =\frac{1}{2} \int_{0}^{x} e^{(x-t) B} B^{-1}\left[h_{+}(t)-h_{+}(x)\right] d t+\frac{1}{2} \int_{x}^{1} e^{(t-x) B} B^{-1}\left[h_{+}(t)-h_{+}(x)\right] d t \\
& +\frac{1}{2} e^{x B} B^{-2} h_{+}(x)+\frac{1}{2} e^{(1-x) B} B^{-2} h_{+}(x)-B^{-2} h_{+}(x) \\
& =\frac{1}{2} \int_{0}^{x} e^{(x-t) B} B^{-1}\left[h_{+}(t)-h_{+}(x)\right] d t+\frac{1}{2} \int_{x}^{1} e^{(t-x) B} B^{-1}\left[h_{+}(t)-h_{+}(x)\right] d t \\
& -B^{-2} h_{+}(x)+\frac{1}{2} e^{x B} B^{-2}\left[h_{+}(x)-h_{+}(0)\right]+\frac{1}{2} e^{(1-x) B} B^{-2}\left[h_{+}(x)-h_{+}(1)\right] \\
& +\frac{1}{2} e^{x B} B^{-2} h_{+}(0)+\frac{1}{2} e^{(1-x) B} B^{-2} h_{+}(1)=v_{R}\left(h_{+}\right)(x)+v_{S, 0}\left(h_{+}\right)(x)+v_{S, 1}\left(h_{+}\right)(x)
\end{aligned}
$$

with

$$
v_{S, 0}\left(h_{+}\right)(x)=\frac{1}{2} e^{x B} B^{-2} h_{+}(0), \quad v_{S, 1}\left(h_{+}\right)(x)=\frac{1}{2} e^{(1-x) B} B^{-2} h_{+}(1) .
$$

Due to Lemma $1, v_{R, 0}\left(h_{+}\right)$has the following maximal regularity properties

$$
\left\{\begin{array}{c}
v_{R}\left(h_{+}\right) \in C\left([0,1] ; D\left(B^{2}\right)\right) \\
B^{2} v_{R}\left(h_{+}\right) \in C^{\eta}([0,1] ; E)
\end{array}\right.
$$

while for $v_{S, 0}\left(h_{+}\right)$and $v_{S, 1}\left(h_{+}\right)$we only have

$$
\begin{cases}\left.\left.v_{S, 0}\left(h_{+}\right) \in C(] 0,1\right] ; D\left(B^{2}\right)\right) ; & \left.\left.B^{2} v_{S, 0}\left(h_{+}\right) \in C^{\eta}(] 0,1\right] ; E\right) \\ v_{S, 1}\left(h_{+}\right) \in C\left(\left[0,1\left[; D\left(B^{2}\right)\right) ;\right.\right. & B^{2} v_{S, 1}\left(h_{+}\right) \in C^{\eta}([0,1[; E) .\end{cases}
$$

Remark 2. We have in view the study of the regularity of $u_{-}$on $[-1,0]$ and $w_{+}$on $[0,1]$. Since

$$
\begin{aligned}
& \left.u_{-}(x)=e^{-x B} \alpha_{-}+e^{(1+x) B} \beta_{-}+v\left(g_{-}\right)(x), \quad x \in\right]-1,0[ \\
& \left.w_{+}(x)=e^{x B} \alpha_{+}+e^{(1-x) B} \beta_{+}+v\left(h_{+}\right)(x), \quad x \in\right] 0,1[
\end{aligned}
$$

the singular parts $v_{S,-1}\left(g_{-}\right), v_{S, 0}\left(g_{-}\right), v_{S, 0}\left(h_{+}\right)$and $v_{S, 1}\left(h_{+}\right)$must be associated with the other singular parts of $u_{-}$and $w_{+}$to find necessary and sufficient conditions on the data $\varphi, \psi, f_{-}, f_{+}, g_{-}$and $h_{+}$in order to get optimal regularity for $u_{-}$on $[-1,0]$ and $w_{+}$on $[0,1]$. 


\subsection{Analysis of the Derivatives}

In order to study the regularity of the solution of our problem, we also need to analyze the behaviour of the derivative of $v\left(g_{-}\right)$and $v\left(h_{+}\right)$. We have, for all $\left.x \in\right]-1,0[$

$$
\begin{aligned}
v^{\prime}\left(g_{-}\right)(x) & =\frac{1}{2} \int_{-1}^{x} e^{(x-t) B} g_{-}(t) d t-\frac{1}{2} \int_{x}^{0} e^{(t-x) B} g_{-}(t) d t+\frac{1}{2} B^{-1} g_{-}(x)-\frac{1}{2} B^{-1} g_{-}(x) \\
& =\frac{1}{2} \int_{-1}^{x} e^{(x-t) B} g_{-}(t) d t-\frac{1}{2} \int_{x}^{0} e^{(t-x) B} g_{-}(t) d t
\end{aligned}
$$

then

$$
\begin{aligned}
v^{\prime}\left(g_{-}\right)(0) & =\frac{1}{2} \int_{-1}^{0} e^{-t B} g_{-}(t) d t=\frac{1}{2} \int_{-1}^{0} e^{-t B}\left[g_{-}(t)-g_{-}(0)\right] d t+\frac{1}{2} \int_{-1}^{0} e^{-t B} g_{-}(0) d t \\
& =\frac{1}{2} B^{-1} g_{-}(0)+\frac{1}{2} \int_{-1}^{0} e^{-t B}\left[g_{-}(t)-g_{-}(0)\right] d t=\frac{1}{2} B^{-1} g_{-}(0)+R_{*}\left(g_{-}\right)(0)
\end{aligned}
$$

Similarly, for all $x \in] 0,1[$, one has

$$
v^{\prime}\left(h_{+}\right)(x)=\frac{1}{2} \int_{0}^{x} e^{(x-t) B} h_{+}(t) d t-\frac{1}{2} \int_{x}^{1} e^{(t-x) B} h_{+}(t) d t,
$$

then

$$
\begin{aligned}
v^{\prime}\left(h_{+}\right)(0) & =-\frac{1}{2} \int_{0}^{1} e^{t B} h_{+}(t) d t=\frac{1}{2} B^{-1} h_{+}(0)-\frac{1}{2} \int_{0}^{1} e^{t B}\left[h_{+}(t)-h_{+}(0)\right] d t \\
& =\frac{1}{2} B^{-1} h_{+}(0)+S_{*}\left(h_{+}\right)(0),
\end{aligned}
$$

and

$$
\begin{aligned}
v^{\prime}\left(h_{+}\right)(1) & =\frac{1}{2} B^{-1} h_{+}(1)+\frac{1}{2} \int_{0}^{1} e^{(1-t) B}\left[h_{+}(t)-h_{+}(1)\right] d t \\
& =\frac{1}{2} B^{-1} h_{+}(1)+S_{*}\left(h_{+}\right)(1) .
\end{aligned}
$$

\section{Study of the Regularity of the Solution of Problem $\left(P_{1 A}\right)$}

\subsection{Necessary Conditions on the Transmission Data}

Assume that $u_{-}$and $w_{+}$are strict solutions, that is $u_{-} \in C([-1,0] ; D(A)) \cap C^{2}([-1,0] ; E)$ and $w_{+} \in C([0,1] ; D(A)) \cap C^{2}([0,1] ; E)$, 
then

$$
\varphi=u_{-}(0)-w_{+}(0) \in D(A)
$$

and for any $\tau_{1} \in[-1,0]$

$$
\left(u_{-}\right)^{\prime}\left(\tau_{1}\right)=\lim _{\tau^{\prime} \rightarrow \tau_{1}} \frac{u_{-}\left(\tau_{1}\right)-u_{-}\left(\tau^{\prime}\right)}{\tau_{1}-\tau^{\prime}} \in \overline{D(A)}
$$

we also deduce that

$$
\left(u_{-}\right)^{\prime \prime}\left(\tau_{1}\right) \in \overline{D(A)} .
$$

Similarly, for any $\tau_{2} \in[0,1],\left(w_{+}\right)^{\prime}\left(\tau_{2}\right),\left(w_{+}\right)^{\prime \prime}\left(\tau_{2}\right) \in \overline{D(A)}$. It follows that

$$
\left\{\begin{array}{c}
\left(u_{-}\right)^{\prime \prime}(0)=g_{-}(0)-A u_{-}(0) \in \overline{D(A)}, \\
\left(w_{+}\right)^{\prime \prime}(0)=h_{+}(0)-A w_{+}(0) \in \overline{D(A)} .
\end{array}\right.
$$

Using the first transmission condition

$$
\varphi=u_{-}(0)-w_{+}(0) \in D(A)
$$

one has

$$
\left(u_{-}\right)^{\prime \prime}(0)=g_{-}(0)-A u_{-}(0)=g_{-}(0)-A \varphi-A w_{+}(0)=g_{-}(0)-A \varphi-h_{+}(0)+\left(w_{+}\right)^{\prime \prime}(0),
$$

from which we deduce the first following necessary compatibility condition

$$
h_{+}(0)-g_{-}(0)+A \varphi=\left(w_{+}\right)^{\prime \prime}(0)-\left(u_{-}\right)^{\prime \prime}(0) \in \overline{D(A)} \text {. }
$$

The second transmission condition gives

$p_{-}\left(u_{-}\right)^{\prime}(0)-p_{+}\left(w_{+}\right)^{\prime}(0)=\psi+q A u_{-}(0)=\psi+q\left[A u_{-}(0)-g_{-}(0)\right]+q g_{-}(0) \in \overline{D(A)}$

which implies the second following necessary compatibility condition

$$
p_{-}\left(u_{-}\right)^{\prime}(0)-p_{+}\left(w_{+}\right)^{\prime}(0)-q\left[A u_{-}(0)-g_{-}(0)\right]=\psi+q g_{-}(0) \in \overline{D(A)} \text {. }
$$

The two conditions (35) - (36) are equivalent to

$$
\left\{\begin{array}{l}
\psi+q g_{-}(0) \in \overline{D(A)} \\
\psi+q\left[h_{+}(0)+A \varphi\right] \in \overline{D(A)}
\end{array}\right.
$$

due to the identity

$$
h_{+}(0)-g_{-}(0)+A \varphi=\frac{1}{q}\left[\psi+q h_{+}(0)+q A \varphi\right]-\frac{1}{q}\left[\psi+q g_{-}(0)\right] \in \overline{D(A)} .
$$

In the case $\psi=0$ and $\varphi=0$, the necessary conditions on the transmission data are

$$
g_{-}(0) \in \overline{D(A)}, \quad h_{+}(0) \in \overline{D(A)} .
$$




\subsection{Analysis of $u_{-}(x)$ Near -1}

Assume the necessary condition $f_{-} \in D\left(B^{2}\right)=D(A)$. Recall that

$$
u_{-}(x)=e^{-x B} \alpha_{-}+e^{(1+x) B} \beta_{-}+v\left(g_{-}\right)(x),
$$

where, due to (23), one has

$$
\beta_{-}=-e^{B} \alpha_{-}-v\left(g_{-}\right)(-1)+f_{-} .
$$

Then the behaviour of $B^{2} u_{-}(x)$ near -1 is the same as that of the function

$$
e^{(1+x) B} B^{2}\left[-v\left(g_{-}\right)(-1)+f_{-}\right]+B^{2} v\left(g_{-}\right)(x) .
$$

One has

$$
\begin{aligned}
v\left(g_{-}\right)(-1) & =\frac{1}{2} \int_{-1}^{0} e^{(t+1) B} B^{-1} g_{-}(-1) d t+\frac{1}{2} \int_{-1}^{0} e^{(t+1) B} B^{-1}\left[g_{-}(t)-g_{-}(-1)\right] d t \\
& =-\frac{1}{2} B^{-2} g_{-}(-1)+R\left(g_{-}\right)(-1)
\end{aligned}
$$

and the term $R\left(g_{-}\right)(-1)$ is regular since

$$
B^{2}\left[R\left(g_{-}\right)(-1)\right] \in D_{B}(\eta ;+\infty),
$$

see Lemma 1.

Now from the study and the results on the behaviour of $B^{2} v\left(g_{-}\right)(x)$ near -1 , see (31), one concludes that the behaviour of $B^{2} u_{-}(x)$ near -1 is the same as that of

$$
e^{(1+x) B}\left[\frac{1}{2} g_{-}(-1)+B^{2} f_{-}\right]+\frac{1}{2} e^{(x+1) B} g_{-}(-1)=e^{(1+x) B}\left[g_{-}(-1)+B^{2} f_{-}\right] .
$$

Due to Lemma $1, B^{2} u_{-}($.$) has the following maximal regularity properties near -1$

$$
\left\{\begin{array}{l}
B^{2} u_{-}(.) \in C\left(\left[-1,0[; E) \text { iff }\left[g_{-}(-1)+B^{2} f_{-}\right] \in \overline{D\left(B^{2}\right)}=\overline{D(B)}\right.\right. \\
B^{2} u_{-}(.) \in C^{\eta}\left(\left[-1,0[; E) \text { iff }\left[g_{-}(-1)+B^{2} f_{-}\right] \in D_{B}(\eta,+\infty) .\right.\right.
\end{array}\right.
$$

\subsection{Analysis of $w_{+}$Near 1}

Let us assume the necessary condition $f_{+} \in D(B)$. Recall that

$$
w_{+}(x)=e^{x B} \alpha_{+}+e^{(1-x) B} \beta_{+}+v\left(h_{+}\right)(x),
$$

then the behaviour of

$$
B^{2} w_{+}(x)=B^{2}\left[e^{x B} \alpha_{+}+e^{(1-x) B} \beta_{+}+v\left(h_{+}\right)(x)\right],
$$

near 1 is the same as that of

$$
B^{2} e^{(1-x) B} \beta_{+}+v\left(h_{+}\right)(x) .
$$


One has seen in (30), (34) that

$$
\begin{aligned}
\beta_{+} & =-e^{B} \Delta^{-1}\left[\left(-p_{-}\left(I+e^{2 B}\right)+q B\left(I-e^{2 B}\right)\right) F_{*}+\left(I-e^{2 B}\right) G_{*}\right] \\
& +B^{-1} v^{\prime}\left(h_{+}\right)(1)-B^{-1} f_{+},
\end{aligned}
$$

with

$$
v^{\prime}\left(h_{+}\right)(1)=\frac{1}{2} B^{-1} h_{+}(1)+S_{*}\left(h_{+}\right)(1) .
$$

Since the behaviour of $B^{2} v\left(h_{+}\right)(x)$ near 1 is that of $\frac{1}{2} e^{(1-x) B} h_{+}(1)$, as mentioned in (33), it follows that the behaviour of $B^{2} w_{+}(x)$ near 1 is that of

$$
-e^{(1-x) B}\left(\frac{1}{2} h_{+}(1)+B f_{+}\right)+\frac{1}{2} e^{(1-x) B} h_{+}(1)=-e^{(1-x) B} B f_{+} .
$$

Due to Lemma $1, B^{2} w_{+}($.$) has the following maximal regularity near 1$

$$
\left\{\begin{array}{l}
\left.\left.B^{2} w_{+}(.) \in C(] 0,1\right] ; E\right) \text { iff } f_{+} \in D(B) \text { et } B f_{+} \in \overline{D(B)} \\
\left.\left.B^{2} w_{+}(.) \in C^{\eta}(] 0,1\right] ; E\right) \text { iff } B f_{+} \in D_{B}(\eta,+\infty) .
\end{array}\right.
$$

\subsection{Analysis of $u_{-}$at the Interface 0}

The behaviour of

$$
B^{2} u_{-}(x)=B^{2} e^{-x B} \alpha_{-}+B^{2} e^{(1+x) B} \beta_{-}+B^{2} v\left(g_{-}\right)(x),
$$

near 0 is the same as that of

$$
B^{2} e^{-x B} \alpha_{-}+B^{2} v\left(g_{-}\right)(x),
$$

and due to (32), it is the same as that

$$
B^{2} e^{-x B} \alpha_{-}+\frac{1}{2} e^{-x B} g_{-}(0) .
$$

Now, in virtue of $(27)$, one has

$$
\begin{aligned}
\alpha_{-} & =[\Delta(B)]^{-1}\left[p_{+}\left(I-e^{2 B}\right) F_{*}-\left(I+e^{2 B}\right) G_{*}\right] \\
& =[\Delta(B)]^{-1}\left[p_{+} F_{*}-G_{*}\right]-e^{2 B}[\Delta(B)]^{-1}\left[p_{+} F_{*}+G_{*}\right] .
\end{aligned}
$$

Write

$$
\begin{aligned}
\Delta(B) & =p_{+}\left(I-e^{2 B}\right)^{2}+p_{-}\left(I+e^{2 B}\right)^{2}-q B\left(I-e^{2 B}\right)\left(I+e^{2 B}\right) \\
& =p_{+} I+p_{-} I-q B+T_{*},
\end{aligned}
$$

where, clearly $T_{*}$ is a very regularizing operator. Then, it suffices to analyze

$$
B^{2} e^{-x B}[\Delta(B)]^{-1}\left[\left(p_{+} F_{*}-G_{*}\right)+\frac{1}{2} B^{-2}\left(p_{+} I+p_{-} I-q B\right) g_{-}(0)\right],
$$

near 0 . First, let us evaluate the term

$$
\left(p_{+} F_{*}-G_{*}\right)+\frac{1}{2} B^{-2}\left(p_{+} I+p_{-} I-q B\right) g_{-}(0),
$$


by using the expressions in (24). One has

$$
\begin{aligned}
F_{*} & =\varphi-e^{B} f_{-}-e^{B} B^{-1} f_{+}+e^{B} v\left(g_{-}\right)(-1)-v\left(g_{-}\right)(0)+e^{B} v^{\prime}\left(h_{+}\right)(1)+v\left(h_{+}\right)(0) \\
& =\left[\varphi-v\left(g_{-}\right)(0)+v\left(h_{+}\right)(0)\right]+R_{*}=\varphi+\frac{1}{2} B^{-2} g_{-}(0)-\frac{1}{2} B^{-2} h_{+}(0)+R_{* *},
\end{aligned}
$$

and

$$
\begin{aligned}
G_{*} & =B^{-1} \psi-\left(p_{-}+q B\right) e^{B} f_{-}+p_{+} e^{B} B^{-1} f_{+}+\left(p_{-}+q B\right) e^{B} v\left(g_{-}\right)(-1) \\
& -p_{+} e^{B} B^{-1} v^{\prime}\left(h_{+}\right)(1)-p_{-} B^{-1} v^{\prime}\left(g_{-}\right)(0)+p_{+} B^{-1} v^{\prime}\left(h_{+}\right)(0)-q B v\left(g_{-}\right)(0) \\
& =B^{-1} \psi-\frac{1}{2} p_{-} B^{-2} g_{-}(0)+\frac{1}{2} p_{+} B^{-2} h_{+}(0)+\frac{1}{2} q B^{-1} g_{-}(0)+S_{*} .
\end{aligned}
$$

Then

$$
\begin{aligned}
& \left(p_{+} F_{*}-G_{*}\right)+\frac{1}{2} B^{-2}\left(p_{+} I+p_{-} I-q B\right) g_{-}(0)=p_{+}\left(\varphi+\frac{1}{2} B^{-2} g_{-}(0)-\frac{1}{2} B^{-2} h_{+}(0)\right) \\
& -\left(B^{-1} \psi-\frac{1}{2} p_{-} B^{-2} g_{-}(0)+\frac{1}{2} p_{+} B^{-2} h_{+}(0)+\frac{1}{2} q B^{-1} g_{-}(0)\right) \\
& +\frac{1}{2} B^{-2}\left(p_{+}+p_{-}-q B\right) g_{-}(0)+P_{*} \\
& =-B^{-1}\left(\psi+q g_{-}(0)\right)+p_{+} \varphi+B^{-2}\left[\left(p_{+}+p_{-}\right) g_{-}(0)-p_{+} h_{+}(0)\right]+P_{*} .
\end{aligned}
$$

Now, as $\varphi \in D\left(B^{2}\right)$ and $B^{-2}\left[\left(p_{+}+p_{-}\right) g_{-}(0)-p_{+} h_{+}(0)\right] \in D\left(B^{2}\right)$, the behaviour of

$$
B^{2} e^{-x B}[\Delta(B)]^{-1}\left[\left(p_{+} F_{*}-G_{*}\right)+\frac{1}{2} B^{-2}\left(p_{+}+p_{-}-q B\right) g_{-}(0)\right],
$$

is that of

$$
B^{2} e^{-x B}[\Delta(B)]^{-1} B^{-1}\left(\psi+q g_{-}(0)\right)
$$

The determinant

$$
\Delta(B)=-q B\left(I-e^{2 B}\right)\left(I+e^{2 B}\right) \Delta_{*},
$$

being invertible of inverse

$$
[\Delta(B)]^{-1}=-\frac{1}{q} \Delta_{*}^{-1}\left(I+e^{2 B}\right)^{-1}\left(I-e^{2 B}\right)^{-1} B^{-1}
$$

(where $\Delta_{*}^{-1}\left(I+e^{2 B}\right)^{-1}\left(I-e^{2 B}\right)^{-1}$ is bounded and commutes with $\left.e^{-x B}\right)$, one concludes that the behaviour of $B^{2} u_{-}(x)$ near 0 is that of

$$
e^{-x B} B B^{-1}\left[\psi+q g_{-}(0)\right]=e^{-x B}\left[\psi+q g_{-}(0)\right] .
$$

Then, due to [7], the necessary and sufficient conditon to obtain the regularity of $u_{-}$near 0 depends of the regularity of the term

$$
\left[\psi+q g_{-}(0)\right]
$$

One gets the following Theorem.

Theorem 2. Assume (16). Let $f_{+} \in D\left((-A)^{1 / 2}\right), \varphi \in D(A), \psi \in E$ and let $g_{-} \in$ $C^{\eta}([-1,0] ; E), h_{+} \in C^{\eta}([0,1] ; E)$ with $0<\eta<1$. Then 
1. $u_{-} \in C([-1,0] ; D(A)) \cap C^{2}([-1,0] ; E)$ if and only if

$$
\left[g_{-}(-1)+B^{2} f_{-}\right] \in \overline{D(A)} \text { and } q g_{-}(0)+\psi \in \overline{D(B)}=\overline{D(A)}
$$

2. $A u_{-}(),.\left(u_{-}\right)^{\prime \prime} \in C^{\eta}([-1,0] ; E)$ if and only if

$$
\left[g_{-}(-1)+B^{2} f_{-}\right] \in D_{A}(\eta / 2,+\infty) \text { and } q g_{-}(0)+\psi \in D_{A}(\eta / 2,+\infty) \text {. }
$$

We have used tha fact that

$$
\overline{D(B)}=\overline{D(A)} \quad \text { and } \quad D_{B}(\eta,+\infty)=D_{A}(\eta / 2,+\infty)
$$

\subsection{Study of $w_{+}$at the Interface 0}

Recall that

$$
B^{2} w_{+}(x)=B^{2}\left[e^{x B} \alpha_{+}+e^{(1-x) B} \beta_{+}+v\left(h_{+}\right)(x)\right]
$$

where

$$
\alpha_{+}=[\Delta(B)]^{-1}\left[-p_{-}\left(I+e^{2 B}\right)+q B\left(I-e^{2 B}\right)\right] F_{*}-\left(I-e^{2 B}\right) G_{*},
$$

(see (28)). Therefore, the behaviour of $B^{2} w_{+}(x)$ near 0 is the same as that of

$$
B^{2} e^{x B} \alpha_{+}+B^{2} v\left(h_{+}\right)(x) .
$$

Now as (see (33)) the behaviour of $B^{2} v\left(h_{+}\right)(x)$ near 0 is that of $\frac{1}{2} e^{x B} h_{+}(0)$ and

$$
\Delta(B)=p_{+} I+p_{-} I-q B+T_{*},
$$

it suffices to analyze the behaviour of

$$
B^{2} e^{x B}[\Delta(B)]^{-1}\left[\left(-p_{-} I+q B\right) F_{*}-G_{*}-\frac{1}{2}\left(p_{+} I+p_{-} I-q B\right) B^{-2} h_{+}(0)\right],
$$

where as we have seen

$$
\left\{\begin{array}{l}
F_{*}=\varphi+\frac{1}{2} B^{-2} g_{-}(0)-\frac{1}{2} B^{-2} h_{+}(0)+R_{* *} \\
G_{*}=B^{-1} \psi-\frac{1}{2} p_{-} B^{-2} g_{-}(0)+\frac{1}{2} p_{+} B^{-2} h_{+}(0)+\frac{1}{2} q B^{-1} g_{-}(0)+S_{*}
\end{array}\right.
$$

with regular terms $R_{* *}$ and $S_{*}$. Then

$$
\begin{aligned}
& \left(-p_{-}+q B\right) F_{*}-G_{*}-\frac{1}{2}\left(p_{+} I+p_{-} I-q B\right) B^{-2} h_{+}(0) \\
& =\left(-p_{-}+q B\right)\left(\varphi+\frac{1}{2} B^{-2} g_{-}(0)-\frac{1}{2} B^{-2} h_{+}(0)\right) \\
& -\left(B^{-1} \psi-\frac{1}{2} p_{-} B^{-2} g_{-}(0)+\frac{1}{2} p_{+} B^{-2} h_{+}(0)+\frac{1}{2} q B^{-1} g_{-}(0)\right) \\
& +\frac{1}{2}\left(p_{+} I+p_{-} I-q B\right) B^{-2} h_{+}(0)+Q_{*}=-B^{-1}\left[\psi-q B^{2} \varphi+q h_{+}(0)\right]+Q_{*},
\end{aligned}
$$


where $Q_{*} \in D\left(B^{2}\right)$. Therefore, the behaviour of $B^{2} w_{+}(x)$ near 0 is that of

$$
B^{2} e^{x B}[\Delta(B)]^{-1} B^{-1}\left[\psi-q B^{2} \varphi+q h_{+}(0)\right] .
$$

as mentioned in the previous section, see (38). One then deduces that the behaviour of $B^{2} w_{+}(x)$ near 0 is that of

$$
e^{x B}\left[\psi-q B^{2} \varphi+q h_{+}(0)\right]
$$

Then, using again [7], the necessary and sufficient conditon to obtain the regularity of $w_{+}$ near 0 depends on the regularity of

$$
\psi-q B^{2} \varphi+q h_{+}(0) .
$$

We conclude by the following Theorem.

Theorem 3. Assume (16). Let $f_{+} \in D\left((-A)^{1 / 2}\right), \varphi \in D(A)$ and $\psi \in E$ and let $g_{-} \in$ $C^{\eta}([-1,0] ; E), h_{+} \in C^{\eta}([0,1] ; E)$ with $0<\eta<1$. Then

1. $w_{+} \in C([0,1] ; D(A)) \cap C^{2}([0,1] ; E)$ if and only if

$$
(-A)^{1 / 2} f_{+} \in \overline{D(A)} \text { and } \psi-q B^{2} \varphi+q h_{+}(0) \in \overline{D(A)},
$$

2. $\left.A w_{+}(),.\left(w_{+}\right)^{\prime \prime} \in C^{\eta}[0,1] ; E\right)$ if and only if $(-A)^{1 / 2} f_{+} \in D_{A}(\eta / 2,+\infty)$ and $\psi-$ $q B^{2} \varphi+q h_{+}(0) \in D_{A}(\eta / 2,+\infty)$.

By observing that

$$
\frac{1}{q}\left[\psi-q B^{2} \varphi+q h_{+}(0)\right]-\frac{1}{q}\left[\psi+q g_{-}(0)\right]=h_{+}(0)-g_{-}(0)+A \varphi,
$$

one deduce the complete Theorem announced in the Introduction.

\section{Going Back to the Concrete Example}

Let us go back to our concrete limiting problem

$$
\left\{\begin{array}{l}
\left.\Delta_{(x, y)} u_{-}=g_{-} \text {in }\right]-1,0[\times \Omega \\
\left.\Delta_{(x, y)} w_{+}=h_{+} \text {in }\right] 0,1[\times \Omega
\end{array}\right.
$$

with the boundary conditions

$$
\left\{\begin{array}{l}
\left.u_{-}=0 \text { on }\right]-1,0[\times \Gamma \\
\left.w_{+}=0 \text { on }\right] 0,1[\times \Gamma \\
u_{-}=f_{-} \text {on }\{-1\} \times \Omega \\
u=f_{-} \text {on }\{-1\} \times \Omega \\
p_{+} \frac{\partial w_{+}}{\partial x}=0 \text { on }\{1\} \times \Omega
\end{array}\right.
$$

and the transmission conditions

$$
\left\{\begin{array}{l}
w_{+}=u_{-} \text {on }\{0\} \times \Omega \\
p_{-} \frac{\partial u_{-}}{\partial x}-p_{+} \frac{\partial w_{+}}{\partial x}=q \Delta_{y} u_{-}+q g_{0} \text { on }\{0\} \times \Omega .
\end{array}\right.
$$


Here $\Omega$ is a bounded domain of $\mathbb{R}^{n}, n \geqslant 1$, with a regular boundary $\Gamma$.

In view to illustrate our abstract analysis, we are going, in this section, to explicit and interpret our impedance compatibility conditions

$$
\begin{gathered}
\left\{\begin{array}{l}
q g_{-}(0)+\psi \in \overline{D(A)} \\
h_{+}(0)-g_{-}(0)+A \varphi \in \overline{D(A)},
\end{array}\right. \\
\left\{\begin{array}{l}
q g_{-}(0)+\psi \in D_{A}(\eta / 2,+\infty) \\
h_{+}(0)-g_{-}(0)+A \varphi \in D_{A}(\eta / 2,+\infty),
\end{array}\right.
\end{gathered}
$$

in the case of the following vector valued Banach spaces

$$
C\left([-1,0] ; C_{0}^{\beta}(\bar{\Omega})\right) \text { and } C\left([0,1] ; C_{0}^{\beta}(\bar{\Omega})\right),
$$

provided that

$$
g_{-} \in C^{\eta}\left([-1,0] ; C_{0}^{\beta}(\bar{\Omega})\right) \text { and } h_{+} \in C\left([0,1] ; C_{0}^{\beta}(\bar{\Omega})\right)
$$

and all the compatibility boundary conditions are satisfied.

Consider the following operator defined in $C_{0}^{\beta}(\bar{\Omega})$ as

$$
\left\{\begin{array}{l}
D(A)=\left\{v \in C^{2}(\bar{\Omega}): v, \Delta_{y} v \in C_{0}^{\beta}(\bar{\Omega})\right\} \\
{[A v](y)=\Delta_{y} v(y) .}
\end{array}\right.
$$

Then $A$ verifes (16) and $\overline{D(A)}$ coincides with the well known little Hölder continuous functions space $h_{0}^{\beta}(\bar{\Omega})$, see [12, p. 497].

Let us point out that the boundary condition in space $C_{0}^{\beta}(\bar{\Omega})$ is essential. Otherwise the estimate in (16) is not verified, see [10, p. 110, Example 3.1.33].

One also has

$$
D(A)=D_{A}(1+\beta / 2,+\infty)=\left\{v \in C^{2+\beta}(\bar{\Omega}): v=\Delta_{y} v=0 \text { on } \partial \Omega\right\} ;
$$

see [10, p. 110, Corollary 3.1.32 and Corollary 3.1.35]. The interpolation $D_{A}(1+\beta / 2,+\infty)$ is intended in the Banach space $C_{0}(\bar{\Omega})$. Therefore, in our subspace $E=C_{0}^{\beta}(\bar{\Omega}) \subset C_{0}(\bar{\Omega})$, one has

$$
\begin{aligned}
D_{A}(\eta / 2,+\infty) & =\left(D(A), C_{0}^{\beta}(\bar{\Omega})\right)_{1-\eta / 2,+\infty} \\
& =\left(D(A), C_{0}^{\beta}(\bar{\Omega})\right)_{1-\eta / 2,+\infty} \\
& =\left(\left\{v \in C^{2+\beta}(\bar{\Omega}): v=\Delta v=0 \text { on } \partial \Omega\right\}, C_{0}^{\beta}(\bar{\Omega})\right)_{1-\eta / 2,+\infty} \\
& =\left(C_{0}^{\beta}(\bar{\Omega}),\left\{v \in C^{2+\beta}(\bar{\Omega}): v=\Delta v=0 \text { on } \partial \Omega\right\}\right)_{\eta / 2,+\infty} \\
& =C_{0}^{\beta+\eta / 2[2+\beta-\beta]}(\bar{\Omega}) \\
& =C_{0}^{\beta+\eta}(\bar{\Omega}),
\end{aligned}
$$

see [10, p. 31, Corollary 1.2.18].

The conditions $\psi=q g_{0} \in E, f_{-} \in D(A)$ become

$$
\left\{\begin{array}{l}
q g_{0} \in C_{0}^{\beta}(\bar{\Omega}) \\
f_{-} \in\left\{v \in C^{2}(\Omega): v, \Delta_{y} v \in C_{0}^{\beta}(\bar{\Omega})\right\}
\end{array}\right.
$$


and $A f_{-}-g_{-}(-1), q g_{-}(0)+\psi, q h_{+}(0)+\psi \in \overline{D(A)}$ signifies

$$
\left\{\begin{array}{l}
y \longmapsto \Delta_{y} f_{-}(y)-g_{-}(-1, y) \in h_{0}^{\beta}(\bar{\Omega}) \\
y \longmapsto g_{-}(0, y)+g_{0}(y) \in h_{0}^{\beta}(\bar{\Omega}) \\
y \longmapsto h_{+}(0, y)+g_{0}(y) \in h_{0}^{\beta}(\bar{\Omega}),
\end{array}\right.
$$

similarly $A f_{-}-g_{-}(-1), q g_{-}(0)+\psi, q h_{+}(0)+\psi \in D_{A}(\eta / 2,+\infty)$ mean

$$
\left\{\begin{array}{l}
y \longmapsto \Delta_{y} f_{-}(y)-g_{-}(-1, y) \in C_{0}^{\beta+\eta}(\bar{\Omega}) \\
y \longmapsto g_{-}(0, y)+g_{0}(y) \in C_{0}^{\beta+\eta}(\bar{\Omega}) \\
y \longmapsto h_{+}(0, y)+g_{0}(y) \in C_{0}^{\beta+\eta}(\bar{\Omega}) .
\end{array}\right.
$$

Note that $C_{0}^{\beta+\eta}(\bar{\Omega}) \subset h_{0}^{\beta}(\bar{\Omega})$.

One can conclude by the following result

Theorem 4. Assume that $g_{-} \in C^{\eta}\left([-1,0] ; C_{0}^{\beta}(\bar{\Omega})\right), h_{+} \in C\left([0,1] ; C_{0}^{\beta}(\bar{\Omega})\right)$ and

$$
q g_{0} \in C_{0}^{\beta}(\bar{\Omega}), f_{-} \in\left\{v \in C^{2}(\Omega): v, \Delta_{y} v \in C_{0}^{\beta}(\bar{\Omega})\right\}:=C_{0,0}^{2+\beta}(\bar{\Omega}) .
$$

Then there exists a unique solution u of Problem (39) - (41) defined as

$$
\begin{aligned}
]-1,0[\cup] 0,1[ & \longrightarrow \\
x & \left.\longmapsto u(x, .)=\left\{\begin{array}{l}
C_{0}^{\beta}(\bar{\Omega}) \\
\left.w_{+}(x, .) \text { on }\right]-1,0[
\end{array}\right] \text { on }\right] 0,1[
\end{aligned}
$$

such that

1. $u_{-} \in C\left([-1,0] ; C_{0,0}^{2+\beta}(\bar{\Omega})\right) \cap C^{2}\left([-1,0] ; C_{0}^{\beta}(\bar{\Omega})\right), w_{+} \in C\left([0,1] ; C_{0,0}^{2+\beta}(\bar{\Omega})\right) \cap$ $C^{2}\left([0,1] ; C_{0}^{\beta}(\bar{\Omega})\right)$ if and only if

$$
\left\{\begin{array}{l}
y \longmapsto \Delta f_{-}(y)-g_{-}(-1, y) \in h_{0}^{\beta}(\bar{\Omega}) \\
y \longmapsto g_{-}(0, y)+g_{0}(y) \in h_{0}^{\beta}(\bar{\Omega}), \\
y \longmapsto h_{+}(0, y)+g_{0}(y) \in h_{0}^{\beta}(\bar{\Omega}),
\end{array}\right.
$$

2. $\Delta_{y} u_{-}, \frac{\partial^{2} u_{-}}{\partial x^{2}} \in C^{\eta}\left([-1,0] ; C_{0}^{\beta}(\bar{\Omega})\right), \Delta_{y} w_{+}(),. \frac{\partial^{2} w_{+}}{\partial x^{2}} \in C^{\eta}\left([0,1] ; C_{0}^{\beta}(\bar{\Omega})\right)$ if and only if

$$
\left\{\begin{array}{l}
y \longmapsto \Delta_{y} f_{-}(y)-g_{-}(-1, y) \in C_{0}^{\beta+\eta}(\bar{\Omega}) \\
y \longmapsto g_{-}(0, y)+g_{0}(y) \in C_{0}^{\beta+\eta}(\bar{\Omega}) \\
y \longmapsto h_{+}(0, y)+g_{0}(y) \in C_{0}^{\beta+\eta}(\bar{\Omega}) .
\end{array}\right.
$$

\section{References}

1. Grisvard P. Spazi di Tracce e Applicazioni. Rendiconti di Matematica, série VI, 1972, vol. 5, no. 4, pp. 657-729. 
2. Krasucki F., Lenci S. Analysis of Interfaces of Variable Stiffness, International Journal of Solids and Structures, 2000, vol. 37, pp. 3619-3632. DOI: 10.1016/S0020-7683(99)00072-4

3. Krasucki F., Lenci S. Yield Design of Bonded Joints. European Journal of Mechanics A/Solids, 2000, vol. 19, issue 4, pp. 649-667. DOI: 10.1016/S0997-7538(00)00173-X

4. Geymonat G., Krasucki F., Lenci S. Mathematical Analysis of a Bonded Joint with a Soft Thin Adhesive. Math. Mech. Solids, 1999, vol. 4, no. 2, pp. 201-225. DOI: $10.1177 / 108128659900400204$

5. Belhamiti O., Labbas R., Lemrabet K., Medeghri A. Transmission Problems in a Thin Layer Set in the Framework of the Hölder Spaces, Resolution and Impedance. Journal of Mathematical Analysis and Applications, 2009, vol. 358, pp. 457-484. DOI: $10.1016 /$ j.jmaa.2009.05.010

6. Dore A., Favini A., Labbas R., Lemrabet K. An Abstract Transmission Problem in a Thin Layer, I: Sharp Estimates. Journal of Functional Analysis, 2011, vol. 261, pp. 1865-1922. DOI: $10.1016 /$ j.jfa.2011.05.021

7. Sinestrari E. On the Abstract Cauchy Problem of Parabolic Type in Space of Continuous Functions. J. Math. Anal. App., 1985, vol. 66, pp. 16-66. DOI: 10.1016/0022-247X(85)90353-1

8. Dore G., Venni A. $H^{\infty}$ Functional Calculus for Sectorial and Bisectorial Operators. Studia Math., 2005, vol. 166, pp. 221-241. DOI: 10.4064/sm166-3-2

9. Haase M. The Functional Calculus for Sectorial Operators and Similarity Methods. Thesis, Universität Ulm, Germany, 2003.

10. Lunardi A. Analytic Semigroups and Optimal Regularity in Parabolic Problems. Birkhäuser, 1995 .

11. Cartan H. Théorie Elémentaire des Fonctions Analytiques d'une ou Plusieurs Variables Complexes. Paris, Hermann, 1961.

12. Campanato S. Generation of Analytic Semigroups by Elliptic Operators of Second Order in Hölder Spaces. Annal. Sc. Norm. Super. Pisa Cl. Sci, 1981, vol. 4, no. 8 (3), pp. 495-512.

13. Da Prato G., Grisvard P. Sommes d'opérateurs linéaires et équations différentielles opérationnelles. J. Math. Pures Appl., IX Ser. 54, 1975, no. 3, pp. 305-387.

14. Labbas R. Problèmes aux limites pour une équation différentielle abstraite de type elliptique. Thèse d'état, Université de Nice, 1987.

15. Pazy A. Semigroups of Linear Operators and Applications to Partial Differential Equations. New York, Applied Mathematical Sciences, Springer-Verlag, 1983. DOI: $10.1007 / 978-1-4612-5561-1$

16. Tanabe H. Equations of Evolution. Monographs and Studies in Mathematics 6 . London, San Francisco, Melbourne, Pitman, 1979.

Received January 14, 2015 


\title{
ЭФФЕКТ ВОЗМУЩЕНИЯ ТОНКОГО КЛЕЕВОГО СЛОЯ В НЕКОТОРЫХ КРАЕВЫХ ЗАДАЧАХ ПЕРЕНОСА НА ОСНОВЕ ЭЛЛИПТИЧЕСКИХ ДИФФЕРЕНЦИАЛЬНЫХ УРАВНЕНИЙ
}

\author{
А. Фавини, Р. Лаббас, К. Лемрабе
}

В данной работе рассматривается задача о двух телах, скрепленных тонким клеевым слоем (третий материал) толщины $\delta$. При $\delta$, стремящемся к нулю, получается краевая задача переноса на фиксированной области. Получены новые результаты по исследованию данной задачи в пространствах Гельдера, а именно, явное представление решения. С помощью теории полугрупп и вещественных интерполяционных пространств получены необходимые и достаточные условия на границе раздела при которых существует единственное решение задачи.

Ключевые слова: эллиптическая краевая задача; задача переноса; эффект возмущения; тонкий слой.

Анджело Фавини, кафедра математики, Болонский университет (г. Болонья, Италия), favini@dm.unibo.it.

Раба Лаббас, лаборатория прикладной математики, Университет Гавра (г. Гавр, Франция), Rabah.Labbas@univ-lehavre.fr.

Кеддуа Лемрабе, факультет математики, Университет науки и технологии - Бумедьен (г. Алжир, Алжир), keddourlemrabet@yahoo.fr.

Поступила в редакиию 14 января 2015 г. 